This item is the archived peer-reviewed author-version of:

The neuropsychology of child sexual offending : a systematic review

\title{
Reference:
}

Dilliën Tineke, Goethals Kris, Sabbe Bernard, Brazil Inte.- The neuropsychology of child sexual offending : a systematic review Aggression and violent behavior - ISSN 1359-1789 - Oxford, Pergamon-elsevier science Itd, 54(2020), 101406

Full text (Publisher's DOI): https://doi.org/10.1016/J.AVB.2020.101406

To cite this reference: https://hdl.handle.net/10067/1679490151162165141 
Running head: NEUROPSYCHOLOGY OF CHILD SEXUAL OFFENDING

\section{The Neuropsychology of Child Sexual Offending: A Systematic Review}

Tineke Dillien $^{1,2}$, Kris Goethals ${ }^{1,2}$, Bernard Sabbe ${ }^{1,3}$, Inti A. Brazil ${ }^{1,4,5,6}$

${ }^{1}$ Collaborative Antwerp Psychiatric Research Institute, University of Antwerp, Belgium

${ }^{2}$ University Forensic Centre, Antwerp University Hospital, Belgium

${ }^{3}$ University Psychiatric Centre Duffel, Antwerp University Hospital, Belgium

${ }^{4}$ Donders Institute for Brain, Cognition and Behaviour, Radboud University, Nijmegen, The Netherlands

${ }^{5}$ Forensic Psychiatric Centre Pompestichting, Nijmegen, The Netherlands

${ }^{6}$ Centre for Advances in Behavioural Science, Faculty of Health and Life Sciences, Coventry University, England

\section{Conflict of Interest statement}

All authors declare not to have any financial interest in the results of this study or any other conflict of interest and agree to transfer copyrights if the manuscript is accepted.

\section{Author Note}

Tineke Dillien was supported by a Dehousse mandate of the University of Antwerp (project number 42/FAO20000/6619). Inti A. Brazil was supported by a VENI grant (451-15-014) awarded by the Netherlands Organisation for Scientific Research (NWO). These funding sources had no involvement in the design, data analysis, or interpretation of the results.

Correspondence concerning this article should be addressed to Tineke Dillien, University of Antwerp, Faculty of Medicine and Health Sciences Campus Drie Eiken D.R.320, Universiteitsplein 1, 2610 Wilrijk, Belgium.

E-mail: tineke.dillien@uza.be 


\begin{abstract}
Relatively little research has examined the neuropsychological functioning of child sex offenders. While research has demonstrated that child sex offenders present with neuropsychological deficits, there is a lack of empirically supported insight into the nature of these deficits. Consequently, important questions concerning the neuropsychological functioning of child sex offenders remain unanswered. Firstly, it is not known conclusively which neuropsychological functions are impaired in child sex offenders. Secondly, the existence of unique neuropsychological profiles in subgroups of child sex offenders has not been established. Thirdly, it is unclear whether the identified neuropsychological deficits are specific to child sex offenders, rather than shared by other offender groups. To address these issues, we conducted a systematic review in which studies examining the neuropsychological functioning of child sex offenders were targeted. The results showed that paedophilic and nonpaedophilic child sex offenders present with specific sets of 'core' neuropsychological deficits, of which some are shared among these subgroups of child sex offenders and with nonsexual offenders. Based on these findings, we propose a preliminary model for the neuropsychological characteristics of different subgroups of child sex offenders.
\end{abstract}

Keywords: child sex offending, paedophilic child sex offending, profiles of neuropsychological functions, specificity of neuropsychological impairments 


\section{Introduction}

Sexual offending against children causes great human suffering, affecting many people (Pereda, Guillera, Forns, \& Gomez-Benito, 2009) and impacting adversely on victims and their families (Maniglio, 2009). Finding therapeutic approaches that are effective in treating individuals who have sexually offended against children and in reducing the risk of sexual recidivism is, therefore, of immense importance. Designing effective interventions requires a sound understanding of the biological and environmental factors that are involved in the aetiology and maintenance of sexual offending behaviour, as well as an understanding of the heterogeneity that exists among the perpetrators of sexual offences against children. Although child sexual offenders (CSOs) are regarded as one group by the general public, they are heterogeneous and differ from each other. Acknowledging this heterogeneity, CSOs are often divided in subgroups based on criminological (e.g., offence or victim characteristics) and / or psychological (e.g., the underlying motivation for the sexual offending behaviour) factors (Robertiello \& Terry, 2007). A distinction between paedophilic and nonpaedophilic CSOs is commonly made, based on the presence of deviant sexual preferences. Although child sexual offending is often equated with paedophilia, this is not correct. Only 40 to $50 \%$ of the CSOs have a sexual preference for prepubescent children and seek sexual gratification by molesting children. The remaining 50 to $60 \%$ are primarily sexually attracted to adults, but turn to children for nonsexual reasons (e.g., when faced with negative emotions) when the opportunity presents itself (e.g., when a potential victim is available) (Seto, 2008). Another common distinction divides CSOs into intrafamilial/incestual CSOs versus extrafamilial CSOs, on the basis of victim relatedness. Whereas intrafamilial CSOs only molest children that they are related to by blood or marriage, extrafamilial CSOs molest children outside of their family. In addition to differences in clinical characteristics and offence behaviour, subgroups of CSOs are assumed to differ in their underlying causal processes as well, signalling the need for therapeutic interventions to be tailored to the unique needs of the individual CSOs. In order to find personalised, effective approaches to treatment, understanding the mechanisms that underlie the aetiology and the maintenance of sexual offending behaviour against children, and unravelling how individuals may differ on the underlying components, is essential.

\subsection{Accounts of the Aetiology and Maintenance of Child Sexual Offending}


In the last two decades, various multifactorial theories have been formulated to explain the aetiology of child sexual offending (Ward, Polaschek, \& Beech, 2006). These theories incorporate several causal factors in a comprehensive model and allow for multiple aetiological pathways toward the onset of child sexual offending. Factors that contribute to the aetiology of child sexual offending include adverse developmental experiences, deviant sexual preferences, preoccupation with sex, cognitive distortions, general antisociality, self-regulation problems, social skill deficits, empathy deficits, interpersonal problems, and loneliness (Ward \& Beech, 2017; Ward et al., 2006). Although aetiological and maintaining factors are not necessarily the same, some of the factors that are involved in the aetiology of child sexual offending, have also been linked to the maintenance of this behaviour. These latter factors are identified in the research literature on sexual recidivism and include sexual deviance, general antisociality, impulsivity, and self-regulation deficits (Hanson \& Morton-Bourgon, 2005; Mann, Hanson, \& Thornton, 2010; Thakker \& Ward, 2012).

Although these accounts provide a view on the aetiology and maintenance of child sexual offending behaviour, Ward and colleagues (Ward \& Beech, 2006; Ward \& Fortune, 2016) argued that significant advances could be made in the field by grounding the aetiological and maintaining factors in neuropsychological constructs. According to them, the aetiological and maintaining factors that are identified are mere descriptions of clinical symptomatology and behaviour that make little or no reference to the processes by which they are formed. The main problem with this behaviour-focused approach is that it gives an incomplete view of the contributing biological or cognitive factors associated with sexual offending. For example, only stating that a CSO exhibits cognitive distortions, without clarifying the mechanisms that are involved in this behaviour (e.g., a misinterpretation of social cues or a post-offense strategies that serve to maintain self-esteem), fails to provide a thorough understanding of an individual sexual offender. Ward and Beech (2006), therefore, argued to push beyond the level of clinical phenomenology when explaining sexual offending behaviour and delve into the processes that underlie the behavioural and clinical phenomena. In this regard, they identify neuropsychological processes as having an important explanatory value, since these processes underpin psychological functioning and human behaviour. This emphasis on neuropsychological 
functions is consistent with the recent trend in psychiatry and psychology to conceptualise mental disorders in terms of underlying psychological mechanisms that are instantiated in neurobiology. Central to this modern approach, in which insights from neuroscience are integrated, is the view that psychopathology is linked to disturbances in the neuropsychological functioning of individuals. An understanding of these factors and how they combine for each individual will provide new ways to describe pathological behaviour, including sexual offending behaviour (Brazil, van Dongen, Maes, Mars, \& Baskin-Sommers, 2018; Montague, Dolan, Friston, \& Dayan, 2012; Stephan \& Mathys, 2014).

In this regard, a key question is whether we have sufficient understanding of the neuropsychological dysfunctions that contribute to child sexual offending. More specifically, do we know enough about differences in the neuropsychological processes that are dysfunctional in CSOs, in a way that would allow a distinction between subgroups based on neuropsychological factors in addition to or instead of behaviour? The identification of unique neuropsychological profiles among CSOs would in time enhance our knowledge of the pathogenesis of the various types of child sexual offending behaviours, leading to better treatment programs. Although the neuropsychological functioning of CSOs has yet to attract the research attention it deserves, several neuropsychological domains received some research interest in the literature on child sexual offending. This paper provides an overview of the empirical literature on the neuropsychological dysfunctions that contribute to child sexual offending. Although two systematic review studies on this topic have been carried out in the last decade, the previous review studies left some important questions unanswered. By including new studies and by considering the methodological quality of the included studies, which was not done in the previous review studies, this new review aims to identify key neuropsychological processes that are afflicted in (subgroups of) CSOs. First, as an introduction to this review, we will briefly discuss the previous review studies and the questions that remained unanswered in these studies. Additionally, we will outline the most prominent neurobiological theories of paedophilia. Although child sexual offending does not equate with paedophilia, these theories inspired the literature on the neuropsychological functioning of CSOs. Then, we will present our systematic review of the empirical literature on the neuropsychology of child sexual offending, with a focus on hands-on child 
sexual offences involving physical contact with the victim (e.g., sexual touching, fondling of genitals or breasts, penetration)

\subsection{The Neuropsychological Correlates of Child Sexual Offending}

1.2.1.Previous review studies. Joyal, Beaulieu-Plante, and de Chanterac (2014) were the first to conduct a meta-analysis of all studies on the neuropsychological functioning of sexual offenders, including CSOs until 2011. The analysis included 23 studies, providing data on 1063 sexual offenders (both offenders who sexually assaulted adults and CSOs), 375 nonsexual offenders and 318 healthy controls. Executive functions have received the most attention in this research domain, followed by memory and verbal functioning. The findings showed that sexual offenders exhibit significant neuropsychological impairments. Indeed, sexual offenders performed significantly worse than both healthy controls and nonsexual offenders, when performances on all neuropsychological tasks were lumped together. Also, the results demonstrated that there is great heterogeneity in the types of neuropsychological impairments seen among sexual offenders, indicating the need to distinguish subgroups of sexual offenders and of using neuropsychological tasks that allow a high level of interpretational precision when studying the neuropsychological functioning of sexual offenders. This latter finding offered tentative support for Joyal et al.’s (2014) main hypothesis which stated that offenders who sexually assaulted adults and CSOs show specific neuropsychological profiles of strengths and weaknesses. Unfortunately, however, the profiles could not be completely developed due to the limited number of studies that administered well-designed neuropsychological tasks to homogeneous subgroups of sexual offenders. In an attempt to complement the meta-analysis of Joyal et al. (2014), Adjorlolo and Egbenya (2016) exclusively studied executive functions (EF) in sexual offenders, with a specific focus on CSOs. In this study, EF was defined as a set of discrete cognitive processes such as cognitive flexibility and resistance to distraction that underlie almost all human behaviour. Specifically, the researchers aimed to determine which EF were most frequently impaired in sexual offenders, particularly in CSOs. Also, the researchers wanted to examine whether subgroups of CSOs showed unique patterns of executive dysfunction. To this end, Adjorlolo and Egbenya (2016) reviewed 18 studies that investigated specific executive dysfunctions in adult sexual offenders. All studies appeared in the literature between 1990 and May 2015, with 11 of them focussing on CSOs. In 
line with the results of Joyal et al. (2014), Adjorlolo and Egbenya (2016) demonstrated that CSOs show executive dysfunctions when compared to comparison groups, especially cognitive flexibility deficits and inhibitory control deficits. No differences in executive dysfunctions were found between subgroups of CSOs. This latter finding contrasts with the finding of Joyal et al. (2014), who found within-group variance in the neuropsychological functioning of CSOs and proposed that subgroups of CSOs (e.g., paedophilic versus nonpaedophilic CSOs; intrafamilial versus extrafamilial CSOs) may show specific neuropsychological profiles.

1.2.2. Link with aetiological and maintaining factors. The focus on executive functioning in the literature is consistent with the significance that theoretical accounts attribute to the role of disinhibition and behavioural dysregulation in the aetiology and maintenance of child sexual offending. From Finkelhor's precondition model to the more recently formulated MotivationFacilitation Model of sexual offending, the motivation to commit a sexual offence against a child has been described as a necessary but insufficient condition to produce a sexual offence. The suggestion is that a sexual offence will be committed only when behavioural control is low and self-regulatory mechanisms fail to suppress the desire to sexually abuse a child. The breakdown in self-regulatory mechanisms can be temporary and result from situational factors, such as alcohol intoxication. But, it can also be more permanent as a consequence of trait-like factors, including self-regulation deficits. In addition to being linked to the aetiology of child sexual offending, an inability to control or regulate one's behaviour has also been identified an as important predictor of (sexual) recidivism, with recidivism studies linking self-regulation deficits, lifestyle instability, and impulsivity to an increased risk of sexual reoffending. Other antisocial populations are also characterized by self-regulation deficits, which suggests that the inability to control or regulate one's behaviour is associated with antisocial behaviour in general, rather than being specific for child sexual offending. Accordingly, in the meta-analysis by Joyal et al. (2014) differences in the neuropsychological functioning between CSOs and nonsexual offenders largely disappeared when only executive function tasks were taken into account.

1.2.3. Strengths and shortcomings of the existing review studies. In general, the metaanalysis by Joyal et al. (2014) was influential in raising attention for the importance of considering 
neuropsychological functioning in sexual offenders and for putting forward that offenders who sexually assaulted adults and CSOs have unique profiles of neuropsychological strengths and weaknesses. Because of the limited number of studies investigating specific neuropsychological domains in homogeneous subgroups of sexual offenders, this meta-analysis did, however, not succeed completely in clarifying the nature of the neuropsychological deficits seen in subgroups of sexual offenders such as CSOs. The review by Adjorlolo and Egbenya (2016), that built on the meta-analysis by Joyal et al. (2014), did also not provide a complete overview of the neuropsychological functioning of CSOs. By exclusively focussing on $\mathrm{EF}$ in $\mathrm{CSO}$, other neuropsychological functions were overlooked. A second limitation of the existing review studies is that they do not allow a firm conclusion with regard to neuropsychological differences between subgroups of CSOs, with the studies producing inconsistent results on this issue. Finally, although the meta-analysis by Joyal et al. (2014) suggested that executive dysfunctions are shared between sexual and nonsexual offenders, no definite conclusions concerning neuropsychological differences and similarities between CSOs and other offender groups were drawn in either of the reviews.

Apart from leaving several important questions unanswered, the existing review studies did not take the methodological quality of the included studies into account and treated all studies equally. Since some of the included studies suffered from serious methodological flaws, the findings and the conclusions from these review studies should be viewed with caution. The present systematic review aims to provide new insights in the neuropsychological functioning of CSOs by examining the empirical work on this topic to date and by critically assessing the quality of the included studies. Because most of these studies were inspired by neurobiological theories of paedophilic child sexual offending, these theories are briefly outlined below.

\subsection{Neurobiological Theories of Paedophilic Child Sexual Offending}

Since neurological case reports demonstrated that paedophilic behaviour can occur in the context of frontal and / or temporal brain lesions that are due to physical conditions such as cancer or dementia (Mohnke et al., 2014), researchers started to hypothesise about the significance of these brain areas in the aetiology of paedophilia. This line of thoughts led to controlled neuroimaging studies in paedophilic CSOs that looked for localised brain abnormalities. The resulting models that link 
paedophilia to brain anomalies in specific brain regions were collectively called 'lesion models' by Cantor et al. (2008). Three lesion models are described in the literature (Cantor et al., 2008; Roszyk \& Łukaszewska, 2-011).

1.3.1.The lesion models. The frontal-dysexecutive model posits frontal lobe anomalies in paedophilic CSOs. As the frontal areas are implicated in cognitive control, abnormalities in these brain areas may lead to a dysfunction in the inhibitory systems. As such, this model stresses the contribution of executive dysfunction and behavioural disinhibition to paedophilic sexual offending behaviour. A variant of this model suggests that brain abnormalities are present in one specific area of the prefrontal cortex, namely the orbitofrontal cortex (OFC). The OFC is involved in several cognitive operations, such as the representation of the reward value of different stimuli and the learning of stimulusreinforcements associations (Kringelbach \& Rolls, 2004; Rolls, 2000). Along with other brain structures including the ventral striatum, the OFC is critically involved in reward processing and plays a crucial role in emotional and social behaviour and in decision-making (Kringelbach \& Rolls, 2004; Rolls, 2000). Proponents of the orbitofrontal dysfunction hypotheses refer to literature describing disturbances in the reward-processing circuit in addictive, impulsive and antisocial behaviour (Blum et al., 2000) and suggest that a similar deficit can play a contributing role in sexual offending (Schiffer et al., 2007). A neuroimaging study probed for structural differences in grey matter concentration in paedophilic CSOs $(n=18)$ relative to healthy controls $(n=24)$ (Schiffer et al., 2007). The results indicated grey matter volume reductions in some of the brain regions that form the frontostriatal system, specifically the OFC and the ventral striatum. Also, grey matter differences were observed in brain systems that interact with the frontostriatal system (i.e., the cerebellum). Opposers of this theory have highlighted that the frontal-dysexecutive theory is not specific for paedophilic CSOs. They argue that the model does not explain paedophilia as such, but rather identifies brain anomalies that provoke a general disinhibition of behaviour, potentially resulting in sexual impulsiveness, hypersexuality etc. In this way, the frontal-dysexecutive theory offers more of an explanation for the acting out of antisocial tendencies rather than for the paedophilic disorder itself (Mohnke et al., 2014; Poeppl et al., 2013). 
Another framework, known as the temporal-limbic theory links paedophilia to temporal lobe and limbic damage. The limbic system is heavily involved in regulating emotions and motivations, including basic human drives such as sexual behaviour. Indeed, studies indicate that lesions in the limbic structures may result in hypersexuality as well as in abnormal sexual interests, such as paraphilic preferences (Spinella \& White, 2006). Using different neuroimaging techniques, a number of studies found temporal lobe anomalies in paedophilic CSOs (Mohnke et al., 2014). For example, Schiltz et al. (2007) analysed magnetic resonance imaging (MRI) scans of paedophilic CSOs $(n=15)$ and healthy controls $(n=15)$ to look for structural differences in brain structures that regulate sexual behaviour. As predicted by the temporal-limbic theory, results showed reduced grey matter in the right amygdala and in related structures, such as the hypothalamus and the septal region in paedophilic CSOs. Poeppl et al. (2013) replicated the findings, showing reduced grey matter in the right amygdala in 9 paedophilic CSOs, but relative to 11 nonsexual offenders instead of a healthy control group. As the latter study failed to replicate grey matter decreases in frontal regions found by Schiffer et al. (2007), the result support the notion that frontal lobe abnormalities are linked to general offending, rather than being specific to paedophilic CSOs.

Cohen et al. (2002) integrated the two lesion models in the dual-dysfunction model postulating that both frontal and temporo-limbic abnormalities underlie paedophilia. According to this model, the temporal-limbic abnormalities account for the pattern of deviant and heightened sexual arousal found in paedophilic CSOs. The prefrontal anomalies are in turn associated with the failure to inhibit sexual urges. Whereas Cohen et al. (2002) were the first to explicitly formulate the dual-dysfunction model, other studies also identified both frontal and temporal brain anomalies (Mohnke et al., 2014).

1.3.2. The neurobiological model of Cantor and co-workers. What the lesion models have in common is that they propose that paedophilia is linked to grey matter abnormalities in localised areas of the brain. This notion has been contested by Cantor et al. (2008) who failed to find grey matter anomalies in paedophilic CSOs. Their MRI study, however, showed that paedophilic CSOs suffer from widespread white matter anomalies in the temporal and parietal lobes. Since these findings differentiated between paedophilic CSOs and nonsexual offenders, Cantor and Blanchard (2012) 
concluded that the white matter anomalies are associated with paedophilia itself, whereas the grey matter anomalies found in other neuroimaging studies are related to offending behaviour. Cantor and al. (2008) linked their findings to the neurodevelopmental hypothesis of paedophilia, stating that preor perinatal neurodevelopmental anomalies that affect the brain broadly increase the risk of paedophilia (Blanchard et al., 2002, 2007). In support of this assumption, Cantor and colleagues (e.g., Cantor et al., 2008) refer to studies that demonstrated the presence of other manifestations of neurodevelopmental anomalies in paedophilic CSOs. More specifically, studies showed that paedophilic CSOs have a higher rate of left-handedness, score lower on overall IQ and display poorer academic performance than nonpaedophilic CSOs (Blanchard et al., 2007; Cantor et al., 2004, 2006; Cantor, Klassen, et al., 2005; Cantor, Blanchard, Robichaud, \& Christensen, 2005). These latter findings, however, do not hold for child pornography offenders who demonstrate high rates of paedophilic sexual interests, with studies showing that child pornography offenders are more intelligent and better educated than hands-on CSOs. In light of this, the idea that these nonspecific signs of neurodevelopmental anomalies are characteristic of paedophilia, irrespective of offending behaviour, seems less likely.

Below, we review the research findings concerning the neuropsychological functioning of male CSOs. Specifically, the following questions are addressed:

- Which neuropsychological functions are impaired in CSOs?

- Are there unique profiles of neuropsychological functioning that capture differences within subgroups of CSOs?

Additionally, the specificity of the identified neuropsychological impairments to (subgroups of) CSOs is addressed.

\section{Method}

\subsection{Inclusion and Exclusion Criteria}

In order to answer these research questions, this review includes empirical papers on the neuropsychological functioning of male CSOs. Inclusion criteria for studies were: 
1. Studies should present original data on the neuropsychological functioning of adult male CSOs who committed hands-on offences.

2. Studies should focus on hands-on CSOs in general or on subgroups of hands-on CSOs.

3. Studies should be published in peer reviewed journals in the English language.

Studies were excluded if (a) they were review articles, meta-analyses or case reports; (b) they included both hands-on and hands-off CSOs in the study group, (c) they lacked a comparison group, and (b) full texts were not available.

\subsection{Search Strategy}

Articles on the neuropsychological functioning of CSOs were identified by searching the Web of Science and Medline databases up to October 2018. In the Web of Science combinations of the following key words were used: "child sex offenders", "child molesters", "paedophile”, "paedophilia”, "incest”, "neuropsychological functioning", "neuropsychological”, "cognitive functioning”, and "executive functioning". Truncation was used to include variations of these terms. Mapping these key words to Subject Headings, the Medline search included combinations of the following Subject Headings: "sex offences", "paedophilia", "child abuse, sexual”, "sex offences", "incest”, “neuropsychological tests", "executive function”, “cognitive dysfunction”, “cognition”, and "cognitive function”. Medical Subject Headings (MeSH) searches were performed.

All titles and abstracts were screened according to the inclusion and the exclusion criteria.

Finally, the reference lists of all retained full text articles and review articles were inspected to identify additional relevant publications. Figure 1 shows the flow chart of the search strategy and the selection of studies for this review.

\subsection{Included Studies}

The database searches yielded 16 articles that met the inclusion criteria and 4 additional articles were identified by scanning the reference lists. Thus, a total of 20 articles were included in this literature review. All studies are cross-sectional. The methodological quality of these studies was evaluated using relevant criteria of The Quality Assessment Tool for Observational Cohort and CrossSectional Studies that was delivered by the US National Institute of Health. More specifically, the following criteria were used to assess the methodological quality of the included studies: (1) the 
representativeness of the subjects within the study sample to the population of CSOs, (2) the presence of a sample size calculation, (3) the validity of the method to assess group membership (i.e., the independent variable), (4) the reliability and validity of the used measures of neuropsychological functioning, (5) the similarity of 'baseline' characteristics of the different study groups (i.e., the appropriateness of exclusion criteria or matching criteria), and (6) statistical control of relevant confounders. With respect to neuropsychological functioning, age and IQ / education level should always be considered as potential confounders. The results of the methodological check were used for the interpretation of the results. Results of poor-quality studies were treated more cautiously and disregarded when drawing conclusions about the neuropsychological functioning of CSOs. Table 1 and table 2 summarise the characteristics, as well as the most important threats to the internal validity of these studies.

In the results section, the included studies are arranged by the specific research question that they provide answers to and divided based on the subgroup of CSOs that they are studying.

\section{Results}

\subsection{Which Neuropsychological Functions are Impaired in Child Sex Offenders?}

Six studies examined the neuropsychological functioning of CSOs (Abracen, O'carroll, \& Ladha, 2008; Joyal, Black, \& Dassylva, 2007; Langevin \& Curnoe, 2008; Scott, Cole, McKay, Golden, \& Liggett, 1984; Turner et al., 2018; Young, Justice, \& Erdberg, 2012, see table 1). Three of these studies employed a standardised test battery, more specifically the Halstead-Reitan (HR) test battery (Reitan \& Wolfson, 1985) or the Luria-Nebraska Neuropsychological Test Battery (Golden, 1980). A standardised test battery is composed of a fixed set of tests that are standardised and validated as a whole (Russell, Russell, \& Hill, 2005). The three other studies examined the neuropsychological performance of CSOs by using idiosyncratic combinations of neuropsychological tests. Throughout this review, we will refer to these test batteries as custom test batteries. Four studies used normative data as comparison, one study used healthy controls, and one study used both healthy controls and nonsexual offender controls. Based on the quality assessment, the studies by Young et al. (2012), Scott et al.(1984), Abracen et al. (2008), and Joyal et al. (2007) were rated as being of fair quality. Common methodological flaws in these studies included small sample sizes and insufficient 
control of relevant confounders. The studies by Langevin and Curnoe (2008) and Turner et al. (2018) were considered to be of good quality.

3.1.1.Studies that used a standardised test battery. Both Young et al.(2012) and Langevin and Curnoe (2008) evaluated the performances of CSOs on the HR battery. In the study by Young et al. (2012) only $13 \%$ of the CSOs obtained an impairment index in the impaired range. The Halstead impairment index is a composite score that gives an indication of general brain functioning. This study also showed that the scores of the CSOs on the HR subtests and on the Wisconsin Card Sorting Test (WCST; Heaton, 1981) were typically in the nonclinical range. On the basis of these findings, Young et al. (2012) concluded that CSOs have intact neuropsychological functioning. Langevin and Curnoe (2008) did, however, not confirm this conclusion. In their study, almost $36 \%$ of the CSOs had an impairment index in the pathological range. Although this study showed that the neuropsychological impairment found among sexual offenders was not independent from confounding variables such as intelligence and age, Langevin and Curnoe (2008) took this finding as evidence that a considerable amount of CSOs suffer from neuropsychological impairment. Scott et al. (1984) who administered the Luria battery to 14 CSOs came to a similar finding with $36 \%$ of the CSOs showing a performance pattern indicative of brain dysfunction (i.e., two or more scale scores exceeded a critical value that was corrected for age and education).

3.1.2. Studies that used custom test batteries. The studies that used custom test batteries to evaluate (mainly) frontally and temporally mediated neuropsychological functions, also gave conflicting results. Abracen et al. (2008) assessed attention, verbal memory, abstract reasoning, and problem solving in a group of CSOs, a group of nonviolent nonsexual offenders and a group of healthy controls. Results showed that CSOs scored significantly worse than the healthy controls (but not the offender controls) on the selected tests. The groups no longer differed, however, after controlling for the effects of age, educational level and drinking history. This finding contrasted with the results by Joyal et al. (2007) who conducted a pilot study. Joyal et al. (2007) administered a test battery that included measures of response inhibition and attention, verbal fluency, verbal memory, cognitive flexibility, and visuospatial abilities (i.e., visuospatial integration and visuospatial memory) to a group of sexual offenders who were divided in CSOs and rapists and compared to normative samples of 
similar age and educational level. Except for visuospatial abilities which are mediated by parietaloccipital regions, all neuropsychological functions are frontally-temporally mediated. The results showed that CSOs performed significantly worse than the normative samples on measures of attention, verbal memory, verbal fluency, and response inhibition. No significant differences were found between the CSOs and the normative samples with regard to cognitive flexibility and visuospatial abilities. The authors concluded on the basis of these findings that child sexual offending is linked to fronto-temporal anomalies that are located in the left hemisphere. Also examining frontal lobe functions, Turner et al. (2018) studied response inhibition and impulsive decision-making in CSOs. In this study, a Go/No-Go task and two decision-making tasks, that is the Iowa Gambling Task (IGT; Bechara, Tranel, \& Damasio, 2000) and the Game of Dice Task (GDT; Brand et al., 2005) were administered to CSOs and healthy controls. In light of the hypothesis that inhibitory control and decision-making impairments could be triggered or worsened in the context of emotional or sexual arousing stimuli, the Go/No-Go task and the IGT were modified to include images of nude children and nude adults as stimuli (i.e., as Go and No-Go cues in the Go/No-Go task and as the backside of the cards of the IGT). These stimuli were taken from the Not Real People picture set (Pacific Psychological Assessment Corperation, 2004). The results from both the classical and the modified decision-making task indicated that CSOs exhibit poor decision-making relative to nonoffender controls, although the group differences on the modified task did not survive the correction for multiple testing. Analysis of performances on the modified task also revealed that the CSOs who scored high on indices of paedophilia were the most severely affected in their decision-making abilities by the presence of salient sexual stimuli. In the modified Go/ No Go task, CSOs made more errors of commission than nonoffender controls, indicating that CSOs are less able to inhibit prepotent responses in the presence of highly salient cues.

3.1.3.Interim summary. Taken together, the studies that examined the neuropsychological functioning of CSOs produced some inconsistent findings. Whereas several studies showed that CSOs have neurocognitive impairments, other studies concluded the opposite. These inconsistencies could be due to between-study differences in study samples, study designs, and outcome measures, but also to methodological flaws in the studies. It is, however, also possible that the inconsistencies in the 
research findings are indicative of the heterogeneity in neuropsychological functioning that is thought to exist among CSOs (Joyal et al., 2014). In light of this suggestion, Joyal et al. (2014) highlighted the necessity to divide CSOs into more homogeneous, and clinically relevant, subgroups of CSOs when studying neuropsychological profiles. Studies that tried to identify unique neuropsychological profiles in subgroups of CSOs will be discussed in detail below.

\subsection{Are there Unique Profiles of Neuropsychological Functioning that Capture Differences Within Child Sex Offenders?}

Fourtien studies examined the neuropsychological functioning of subgroups of CSOs (see table 2). All studies studied predetermined subgroups of CSOs, with most studies dividing CSOs on the basis of sexual preferences into paedophilic and nonpaedophilic CSOs (Azizian, Hutton, Hughes, \& Sreenivasan, 2016; Cohen, Nesci, Steinfeld, Haeri, \& Galynker, 2010; Cohen et al., 2002; Eastvold, Suchy, \& Strassberg, 2011; Kärgel et al., 2017; Kruger \& Schiffer, 2011; Langevin, Wortzman, Wright, \& Handy, 1989; Massau et al., 2017; Schiffer \& Vonlaufen, 2011; Suchy, Whittaker, Strassberg, \& Eastvold, 2009a, 2009b; Suchy, Eastvold, Strassberg, \& Franchow, 2014). A very limited number of studies used another way of classifying CSOs and distinguished between incestual and nonincestual CSOs on the basis of victim relatedness (Becerra-García \& Egan, 2014; Langevin, Wortzman, Dickey, Wright, \& Handy, 1988; Langevin, Wortzman, Wright, \& Handy, 1989). Three of these studies used a standardised test battery, while custom test batteries were used in the remaining eleven studies. All studies had some methodological limitations, including uncertain representativeness of the study participants and lack of sample size calculations, but in general the methodological quality of these studies was satisfactory. Based on the quality assessment, the studies by Suchy et al. (2009a), Schiffer and Vonlaufen (2011), Eastvold et al. (2011), Kärgel et al. (2017), Cohen et al.(2002), Massay et al. (2017), Kruger and Schiffer (2011), Azizian et al. (2016), Suchy et al. (2009b), Suchy et al. (2014), and by Becerra-Garcia and Egan (2014) were considered of good quality. The studies by Cohen et al. (2010), Langevin et al. (1989), and Langevin et al. (1988) were considered of low quality because they suffered from major methodological limitations. Cohen et al. (2010) used an invalid method to diagnose paedophilia, making it a real possibility that nonpaedophilic CSOs had been included in the paedophilic sample. The study by Langevin et al. (1989) 
ran the risk of measurement error by using measures that are psychometrically inadequate or less appropriate for our specific research question. More specifically, the Wechsler Memory Scale (WMS; Wechsler, 1945) has important methodological limitations (Prigatano, 1978) and the Differential Aptitude (DAP) Space Relations Test (Bennett, Seashore, \& Wesman, 1982) was not developed for use with clinical populations. The latter study (Langevin et al., 1988) made little or no attempt to control for relevant confounding variables

\subsubsection{The neuropsychological profiles of paedophilic and nonpaedophilic child sexual}

offenders. In accordance with the suggested link between impaired EF, behavioural disinhibition and paedophilic child sexual offending (Cohen et al., 2002), a significant number of studies examined $E F$ in CSOs. One of these studies (Suchy et al., 2009a) assessed EF by calculating a composite score of several EF tasks. Results of this study showed that both paedophilic and nonpaedophilic CSOs demonstrated significantly more impairment in EF than healthy controls. In the other studies, EF was broken down into a number of elemental cognitive processes (Suchy, 2009). These studies more specifically focussed on cognitive flexibility, working memory, and inhibitory control or impulsivity when studying EF profiles, but also investigated the role of attention.

3.2.1.1.Inhibotory control. Regarding inhibitory control, Schiffer and Vonlaufen (2011) found that both paedophilic and nonpaedophilic CSOs show impairments relative to nonsexual offenders and healthy controls. Only the difference between nonpaedophilic CSOs and the two control groups remained significant after Bonferonni corrections. Still, Schiffer and Vonlaufen (2011) concluded that both CSOs groups have difficulties with inhibition. Eastvold et al. (2011) came to the same conclusion when measuring inhibitory control. They more specifically showed that both paedophilic CSOs and nonpaedophilic CSOs scored significantly lower than nonsexual offenders on a measure of inhibition, with paedophilic CSOs demonstrating the greatest impairment. This latter finding was, however, inconsistent with findings of the same study that demonstrated that paedophilic CSOs performed better than nonpaedophilic CSOs with respect to planning and performance accuracy, suggesting better inhibitory abilities in paedophilic than in nonpaedophilic CSOs. In attempting to explain this inconsistency, Eastvold et al. (2011) emphasised that the performance on the timed inhibition tasks was dependent on work pace (and processing speed). Poor performance on these tasks 
could be consequently due to a slow work pace, rather than to a deficit in inhibitory control. A supplementary analysis in which error rates were also considered, showed that this could be the case for paedophilic CSOs who worked slowly, but accurately.

Other studies also questioned whether paedophilic CSOs are characterised by inhibitory control impairments. In a study by Kärgel et al. (2017) paedophilic CSOs, paedophiles who had not engaged in child sexual offending, and healthy controls performed a Go/ No-Go task while being scanned with fMRI. Analysis of the behavioural data of the Go/ No-Go task showed that the nonoffending paedophiles outperformed the paedophiles who had sexually offended against a child. Since both offending paedophiles and nonoffending paedophiles did not differ significantly from healthy controls with respect to the ability to inhibit prepotent responses, these results were interpreted to suggest that nonoffending paedophiles are characterised by superior inhibitory control abilities, rather than that offending paedophiles suffer from impaired inhibitory control. Similarly, in two studies, Cohen and colleagues (Cohen et al., 2010, 2002) showed that paedophilic CSOs are not impulsive, with impulsivity being related to inhibitory control (Eastvold et al., 2011; Schiffer \& Vonlaufen, 2011). The results from the latter study (Cohen et al., 2010) should, however, be interpreted with caution, due to some important methodological limitations (see table 2).

In contrast, the study by Massau et al. (2017) did show inhibitory control deficits both in paedophilic and in nonpaedophilic CSOs. In this study the performances of paedophilic CSOs, nonpaedophilic CSOs, healthy controls, and nonoffending paedophiles were compared on an inhibitory control task. It was found that both groups of CSOs showed impairment in inhibitory control, relative to healthy controls but also to nonoffending paedophiles. These differences, did, however, not survive Bonferroni correction.

3.2.1.2. Cognitive flexibility. Cognitive flexibility is another area that has received attention in empirical work on child sexual offending. Both Schiffer and Vonlaufen (2011) and Eastvold et al. (2011) showed no differences in cognitive flexibility between paedophilic, nonpaedophilic CSOs and nonsexual offenders. When comparing paedophilic and nonpaedophilic CSOs to healthy controls, Schiffer and Vonlaufen found cognitive flexibility deficits in both groups of CSOs, with nonpaedophilic CSOs exhibiting the most profound performance deficits. Whereas nonpaedophilic 
CSOs and healthy controls differed on all outcome measures of the cognitive flexibility task, paedophilic CSOs and healthy controls only differed with respect to the number of perseverative errors. After Bonferroni correction, only the difference between nonpaedophilic CSOs and healthy controls with respect to the number of categories achieved survived Bonferroni correction. In contrast to the methodologically flawed study by Cohen et al. (2010) that described cognitive flexibility impairments in paedophilic CSOs, other studies also failed to find profound cognitive flexibility impairments in paedophilic CSOs. In the study by Cohen et al. (2002) paedophilic CSOs and healthy controls scored equally on measures of cognitive flexibility. Whereas Kruger and Schiffer (2011) did find differences between paedophilic CSO and healthy controls in cognitive flexibility, these differences disappeared when age was entered as a covariate. The study by Massau et al. (2017) even found that paedophilic CSOs performed better than healthy controls, nonpaedophilic CSOs, or nonoffending paedophiles on a cognitive flexibility task.

3.2.1.3.Working memory. Working memory was also assessed. Although working memory is widely studied in the psychological literature, there is no consensus on the definition of working memory and how it should be measured (Aben, Stapert, \& Blokland, 2012). Eastvold et al. (2011) used backward span tasks to assess working memory capacities, and found no differences between paedophilic CSOs, nonpaedophilic CSOs and nonsexual offenders. In the study by Kruger and Schiffer (2011) in which paedophilic CSOs were compared to healthy controls on a span task measuring visuospatial working memory, paedophilic CSOs demonstrated intact spatial working memory capacities. This latter finding was confirmed by Massau et al. (2017) who administered a spatial span task to paedophilic CSOs, nonpaedophilic CSOs, nonoffending paedophiles, and healthy controls. In contrast to the paedophilic CSOs who did not differ from the control groups, nonpaedophilic CSOs performed more poorly than healthy controls on this task, indicating spatial working memory deficits in nonpaedophilic CSOs. It should be noted, however, that the difference between nonpaedophilic CSOs and healthy controls did not survive Bonferroni correction.

3.2.1.4.Attentional functioning. Several studies specifically focussed on the attentional functioning in CSOs. Debate also exists in the literature on this neuropsychological process. It is generally agreed upon that attention is not a unitary concept but contains several different dimensions. 
Different views however exist on how to subdivide attention (Chun, Golomb, \& Turk-Browne, 2011). In order to bypass this debate, we will group the studies according to the tasks that were used to measure attention. Eastvold et al. (2011) used memory span tasks to assess attention, and found no significant differences between paedophilic CSOs, nonpaedophilic CSOs and nonsexual offenders. In the study by Azizian et al. (2016) paedophilic CSOs performed significantly worse than the normative comparison sample on the attention index score of the Repeatable Battery for the Assessment of Neuropsychological Status (RBANS; Randolph, 1998). This index score comprises 2 subtests, one memory span task and one perceptual speed task. The paedophilic CSOs scored equally to the normative sample on the first subtest, but significantly lower on the second one. Similarly, Kruger and Schiffer (2011) found significant differences between paedophilic CSOs and healthy controls on all but one scale of an attention test that emphasises perceptual speed. Although these differences were no longer statistically significant after age was taken into account as a confounding variable, the authors concluded that paedophilic CSOs performed poorly on attention and information processing. In contrast, Cohen et al . (2002) who also used a perceptual speed task, that is the TMT A, found no significant differences between paedophilic CSOs and healthy controls. A second study by the same research group confirmed this latter finding (Cohen et al., 2010). This latter result should, however, be treated with care because of the methodological limitations of the study. Inconsistent findings were also reported when attention was assessed by response selection tasks. Whereas Cohen et al. (Cohen et al., 2002) found no differences between paedophilic CSOs and healthy controls on the Stroop ColorWord Test (Delis, Kaplan, \& Kramer, 2001), their later, lower quality, study showed that paedophilic CSOs scored significantly worse than healthy controls on this task (Cohen et al., 2010).

3.2.1.5.Nonexecutive functions. Although most attention was given to $\mathrm{EF}$, nonexecutive functions were also examined in paedophilic and nonpaedophilic CSOs. In order to study memory processes, Langevin et al. (1989) administered the Wechsler Memory Scale (WMS; Wechsler, 1945) to a group of paedophilic CSOs. The WMS consists several memory tests of which the scores are summed to yield a composite score for memory functioning (i.e., the memory quotient). Since paedophilic CSOs had a total memory quotient within normal limits, Langevin et al. concluded that paedophilic CSOs have intact memory abilities. This study, however, had important methodological 
limitations that could have affected the findings (see table 2). Notwithstanding this, the study by Suchy et al. (2009a) also failed to find differences in the composite scores of auditory and visual memory between paedophilic CSOs, nonpaedophilic CSOs and healthy controls. Schiffer and Vonlaufen (2011) who compared paedophilic CSOs, nonpaedophilic CSOs, nonsexual offenders, and healthy controls on verbal memory and visuospatial memory tasks, confirmed these findings for paedophilic CSOs, but not for nonpaedophilic CSOs. Whereas nonpaedophilic CSOs did not differ from the other groups with respect to visuospatial memory functioning, both nonpaedophilic CSOs and nonsexual offenders performed more poorly than paedophilic CSOs and healthy controls on the verbal memory tasks. In contrast to the studies showing intact verbal memory functioning in paedophilic CSOs, Azizian et al. (2016) demonstrated that paedophilic CSOs obtained significantly lower scores than the normative sample on immediate and delayed memory indices that consist mainly of verbal tasks.

Regarding verbal functioning, Azizian et al. (2016) found paedophilic CSOs to score significantly lower than the normative comparison sample on a verbal fluency task. Similarly, Schiffer and Vonlaufen (2011) demonstrated that paedophilic CSOs, nonpaedophilic CSOs, and nonsexual offenders performed worse than healthy controls on a verbal fluency task, but none of these differences survived Bonferroni correction. In the study by Cohen et al. (2002), no verbal fluency differences between paedophilic CSOs and healthy controls were reported. The lower quality study by Cohen et al. (2010) led to the same conclusion.

Azizian et al. (Azizian et al., 2016) also studied visuo-spatial functioning in paedophilic CSOs. In this study, it was found that paedophilic CSOs scored significantly lower on visuospatial functioning when compared to the normative sample. This finding was contradicted by Langevin et al. (1989) who concluded that paedophilic CSOs had intact visuospatial abilities. However, since this latter study was methodologically flawed, the results of this study should be interpreted cautiously.

One study (Suchy et al., 2009b) focussed on affect recognition abilities and studied both facial and prosodic affect recognition abilities. Since nonpaedophilic CSOs performed more poorly than paedophilic CSOs and healthy controls in a facial affect and a prosodic affect recognition task, the 
authors concluded that nonpaedophilic CSOs are impaired in their ability to recognise other people's emotional states, relative to paedophilic CSOs and healthy controls.

Another cognitive process that caught the attention of researchers who studied paedophilic CSOs is processing speed. Different studies demonstrated that paedophilic CSOs showed slower processing speed than nonpaedophilic CSOs, nonsexual offenders, or healthy controls (Eastvold et al., 2011; Suchy et al., 2009a). On the basis of these studies, it was however not clear how the processing speed weakness in paedophilic CSOs should be interpreted: as a neurocognitive problem or as a reflection of a thoughtful, planful response style that favours accuracy at the expense of speed. A later study confirmed the presence of processing speed weaknesses among paedophilic CSOs relative to nonsexual offender controls or normative data (Suchy et al., 2014). This study, moreover, demonstrated conclusively that the poor processing speed in paedophilic CSOs reflected a fundamental neurocognitive weakness. Because of the important role of white matter pathways in processing speed, Suchy et al.(2014) related their findings to the work of Cantor et al. (2008) who had described extensive white matter abnormalities in paedophilic CSOs.

\subsubsection{The neuropsychological profiles of incestual and nonincestual child sexual}

offenders. In one of the few studies that examined the neuropsychological functioning of incestual CSO, Langevin et al. (1988) used the HR battery. While $13.3 \%$ of the incestual CSO obtained an impairment index in the pathological range, no significant difference was found with the comparison group that was comprised of nonviolent nonsexual offenders. On subtest level, incestual CSO scored significantly more impaired than control subjects on the Rhythm test, the Trail Making test A and B and the Categories test. The methodological limitations of this study, however, limit the confidence in these findings (see table 2).

Both Becerra-Garcia and Egan (2014) and Langevin et al. (1989) studied the neuropsychological functioning of incestual CSOs by using custom test batteries. Becerra-Garcia and Egan (2014) examined executive differences between incestual and nonincestual CSOs and assessed processing speed, cognitive flexibility, executive control, and working memory. Results showed that both groups of CSOs exhibited deficits in processing speed, cognitive flexibility, and executive control relative to healthy controls. With regard to the working memory tasks (i.e., a forward and a backward 
span task), only incestual CSOs showed deficits in comparison with healthy controls. While no differences were found between the two groups of CSOs in processing speed, cognitive flexibility, and executive control, the scores on the backward span task did differentiate between incestual and nonincestual CSOs offenders. Specifically, nonincestual CSOs outperformed incestual CSOs. Langevin et al. (1989) focussed on functions that are controlled by the temporal lobes and administered the WMS and the DAP Space Relations Test to a group of incestual CSOs. Results showed that incestual CSOs have intact visuospatial and memory abilities. However, since the WMS and the DAP Space Relations Test have important psychometric limitations, this conclusion should be regarded as tentative.

3.2.3.Interim summary. Until now, a limited number of studies have been done that tried to identify unique neuropsychological profiles in subgroups of CSOs that are defined on the basis of clinical symptomatology or offence behaviour. These studies were mainly found for paedophilic (versus nonpaedophilic) CSOs and were primarily focussed on EF. Although discrepancies exist between studies, the results generally suggest that paedophilic and nonpaedophilic CSOs present with specific neuropsychological profiles that are distinct but show some overlap as well. Disregarding the studies of poor quality, a tentative model for the neuropsychological deficits of paedophilic and nonpaedophilic CSOs will be proposed in the discussion section.

\section{Discussion}

Therapeutic interventions that are aimed at reducing sexual offending behaviour against children could benefit from a better understanding of the neuropsychological aspects of this behaviour. In order to examine the neuropsychological correlates of this form of offending behaviour, we reviewed the empirical literature to determine 1) which neuropsychological functions are impaired in CSOs and 2) if subgroups of CSOs present with unique profiles of neuropsychological functioning? We additionally were interested in the specificity of the identified neuropsychological impairments for (subgroups of) CSOs.

\subsection{Identifying Neuropsychological Impairments in Child Sexual Offenders}

Six studies have examined the neuropsychological functioning of CSOs, with conflicting 
results. Langevin and Curnoe (2008), Scott et al. (1984), Joyal et al. (2007), and Turner et al. (2018) found that CSOs molesters present with neuropsychological impairments, with the two latter studies showing that CSOs are typified by a limited set of 'core' neuropsychological deficits associated with the modulation of attention, verbal functioning, and the regulation of behaviour. These results do not agree with the studies by Young et al.(2012) and Abracen et al. (2008) that demonstrated no neuropsychological impairments in CSOs compared to healthy controls or nonsexual nonviolent offenders. It is unclear how the disparity in research findings should be interpreted. On the one hand, it is possible that some of the findings were compromised by methodological weaknesses within the studies. Only two studies were assessed as being of good quality. The other studies suffered from methodological limitations that could have affected the findings. On the other hand, it is possible that the diverging results do not contradict each other, but merely confirm the neuropsychological heterogeneity among CSOs that was suggested in the meta-analysis by Joyal et al. (2014). If this latter suggestion is true, different neuropsychological profiles will exist within CSOs.

\subsection{Unique Profiles of Neuropsychological Functioning in Subgroups of Child Sexual Offenders}

In order to explore whether distinct neuropsychological profiles exist among CSOs, we examined studies that investigated the neuropsychological functioning of subgroups of CSOs. All studies divided CSOs into more homogeneous subgroups, with most of them classifying CSOs on the basis of clinical characteristics (i.e., the presence of paedophilia), and a minority classifying CSOs on the basis of victim relatedness. Although these studies produced some inconsistent findings, they support the notion that subgroups of CSOs present with specific neuropsychological profiles. Interestingly, however, this is only true when CSOs are divided on the basis of the presence of paedophilic preferences and not when CSOs are divided on the basis of victim relatedness (Azizian et al., 2016; Becerra-García \& Egan, 2014; Cohen et al., 2010, 2002; Eastvold et al., 2011; Kärgel et al., 2017; Kruger \& Schiffer, 2011; Langevin et al., 1988, 1989; Massau et al., 2017; Schiffer \& Vonlaufen, 2011; Suchy et al., 2014, 2009a, 2009b). The only methodologically adequate study that divided CSOs on the basis of victim relatedness showed little or no differences in neuropsychological profiles between incestual and nonincestual CSOs. Both groups of CSOs were found to demonstrate impairments in processing speed, cognitive flexibility, and executive control relative to healthy 
controls and only differed from each other with respect to working memory (Becerra-García \& Egan, 2014). In contrast, distinct neuropsychological profiles emerged for paedophilic and nonpaedophilic CSOs. In order to delineate the neuropsychological profiles of paedophilic and nonpaedophilic CSOs as accurately as possible, it is necessary to distinguish between studies that used healthy controls and studies that used offender controls. Both study designs have merit but answer different research questions. Whereas the former studies shed light on the neuropsychological impairments that are present in CSOs, the latter studies help to determine the specificity of the identified neuropsychological impairments for CSOs (Eastvold et al., 2011). Only three studies were found that used nonsexual offender controls as a comparison group (Eastvold et al., 2011; Schiffer \& Vonlaufen, 2011; Suchy et al., 2014).

4.2.1.Neuropsychological profiles of paedophilic and nonpaedophilic CSOs. Based on the studies that compared subgroups of CSOs to healthy controls, the following neuropsychological profiles emerged. Paedophilic CSOs show impairment on a composite score for EF. When EF is broken down into various lower-level cognitive processes, paedophilic CSOs have intact visuospatial working memory and intact (superior) cognitive flexibility but show impairments in attention as assessed by perceptual speed tasks. Some studies demonstrate impaired inhibitory control in paedophilic CSOs, but not all studies have found this. Paedophilic CSOs show intact affect recognition skills but demonstrate diminished processing speed and seem to have impaired visuospatial functioning. They have intact visuospatial memory and verbal memory abilities. Whether verbal fluency is impaired, is unclear. Nonpaedophilic CSOs show more extensive and profound executive dysfunctions. In addition to demonstrating an impaired EF composite, they show impaired inhibitory control, cognitive flexibility, and visuospatial working memory. In regard to nonexecutive functions, they exhibit intact processing speed and visuospatial memory skills, but demonstrate impaired verbal memory, verbal fluency, and affect recognition. Figure 2 provides a graphical depiction of a preliminary taxometric model for paedophilic and nonpaedophilic CSOs based on neuropsychological features (see also Brazil et al., 2018). Since empirical findings are still limited and mixed, this model needs to be regarded as tentative and much more research is needed to elaborate these conclusions. Still, it provides a clear overview of the current state of affairs. 
As shown in Figure 2, the neuropsychological profiles of paedophilic and nonpaedophilic CSOs are generally distinct, and can be differentiated on the basis of neuropsychological functions such as processing speed, verbal memory abilities, and affect recognition. The profiles, however, show some overlap as well, with both groups presenting with executive dysfunctions. Although nonpaedophilic CSOs are more broadly and severely impaired in their executive functioning than paedophilic CSOs, both subgroups of CSOs showed inhibitory control impairments. This indicates that paedophilic and nonpaedophilic CSOs have at least some shared neuropsychological features in the form of executive dysfunctions, specifically inhibitory control dysfunction. Being particularly involved in self-regulation and behavioural control, it is reasonable to assume that this dysfunction plays a major role in moving individuals with deviant tendencies toward the commission of a sexual offence. The studies by Massau et al. (2017) and Kärgel et al. (2017), in which inhibitory control was found to differentiate between CSOs and paedophilic individuals who refrain from offending, are in line with this idea.

\subsubsection{The specificity of the neuropsychological impairments for paedophilic and}

nonpaedophilic CSOs. Similar to CSOs, other antisocial populations also show inhibitory control deficits and other executive dysfunctions (Hoaken, Allaby, \& Earle, 2007; Ogilvie, Stewart, Chan, \& Shum, 2011). Executive dysfunctions are thus most likely linked to antisocial, rule breaking behaviour in general, rather than to child sexual offending specifically. The same reasoning applies to verbal memory impairments and facial affect recognition deficits which also have been found in antisocial populations (Wood \& Liossi, 2006). Since these latter dysfunctions are only shown in nonpaedophilic CSOs, it is reasonable to suggest that nonpaedophilic CSOs and nonsexual offenders are neuropsychologically more similar than paedophilic CSOs and nonsexual offenders.

The few studies that compared paedophilic and nonpaedophilic CSOs to nonsexual offenders with respect to cognitive flexibility, working memory (as assessed by backward span tasks), and attentional functioning (as assessed by memory span tasks) provided evidence for the notion that executive dysfunctions are shared among CSOs and with nonsexual offender groups. Notwithstanding the similarities in executive functioning between paedophilic CSOs, nonpaedophilic CSOs and nonsexual offender groups, these studies are also in accordance with the suggestion that 
nonpaedophilic CSOs and nonsexual offenders are more similar in their neuropsychological functioning than paedophilic CSOs and nonsexual offenders. Whereas both nonpaedophilic CSOs and nonsexual offenders show verbal memory impairments, paedophilic CSOs show superior verbal memory capabilities relative to offender controls. Paedophilic CSOs also differ from nonsexual offenders in terms of processing speed, with nonsexual offenders outperforming the paedophilic CSOs.

\subsection{Clinical Implications}

The differences in neuropsychological functioning are on group level and, therefore, probably do not exist for all individual CSOs. When neuropsychological impairments are however present, they have important clinical implications as they may explain why some CSOs show poor treatment response and fail to obtain desired therapeutic results. Offender treatment aims to change maladaptive behaviour and thinking patterns by using interventions that involve self-monitoring, problems solving, weighing up pros and cons of behaviour patterns, etc. Since these interventions place heavy demands on neuropsychological functioning, neuropsychologically impaired CSOs may be hindered in their capacity to benefit from therapy. Thought should be consequently given to how interventions should be modified to meet the needs of CSOs who suffer from neuropsychological impairments that potentially make it difficult to meet therapeutic goals. The necessity to take into account factors that prevent CSOs from profiting from treatment has been described in the responsivity principle. In light of the differences in the neuropsychological profiles, it is reasonable to assume that the difficulties that need to be addressed will differ between paedophilic and nonpaedophilic CSOs. For example,

paedophilic CSOs who suffer from attentional deficits and processing speed difficulties might benefit from having the length and pace of a therapeutic session reduced, whereas nonpaedophilic CSOs who are impaired in their verbal memory functioning might require simplified verbal interactions and the use of memory aids, repetition, and rehearsal. Additionally, since neuropsychological impairments may underlie factors that drive sexual offending, remediating these impairments may be necessary to tackle the risk factors associated with sexual reoffending fundamentally. For example, remediating inhibitory control impairments will increase the capacity to regulate oneself and control one's behaviour. Likewise, remediating affect recognition impairments might be crucial to ameliorate 
difficulties in establishing intimate partner relationships. In this way, enhancing existing treatment programs for CSOs by interventions that address the observed neuropsychological deficits (Baskin-Sommers, Curtin, \& Newman, 2015) could be helpful to improve the effectiveness of sexual offender treatment and reduce the likelihood of further sexual offending behaviour.

\subsection{Limitations and Future Studies}

While the results of the present review help elucidate the neuropsychological characteristics of (different subgroups of) CSOs, it is important to consider that our conclusions were based on a rather limited body of evidence. Moreover, none of the included studies were free of methodological flaws. Although the more recent studies were generally methodologically more rigorous than the older studies, they also suffered from methodological limitations that could have had an impact on the results. Our conclusions should, therefore, be regarded as tentative, and should be further investigated in future studies, highlighting the need of conducting further research on this topic. Future studies should pay sufficient attention to confounding variables and not limit themselves to age and IQ/education. Less obvious characteristics, that have largely been ignored up to now, may affect neuropsychological functioning as well. These potentially relevant variables include substance abuse, comorbid psychiatric diagnoses, and length of imprisonment.

\section{Conclusions}

The results indicate that paedophilic and nonpaedophilic CSOs present with specific neuropsychological profiles that are distinct, while also showing some overlap. Based on available studies, several conclusions were drawn and a preliminary taxonomic model for paedophilic and nonpaedophilic CSOs based on neuropsychological functioning was suggested. A more complete understanding of the neuropsychological factors that contribute to child sexual offending has the potential to move the field forward by offering insight into the mechanistic impairments that play a role in the aetiology and maintenance of sexual offending toward children, ultimately leading to the development of targeted (i.e., personalised) interventions for CSOs in which individual differences in vulnerabilities can be better accounted for. 


\section{References}

Aben, B., Stapert, S., \& Blokland, A. (2012). About the distinction between working memory and short-term memory. Frontiers in Psychology, 3. https://doi.org/10.3389/fpsyg.2012.00301

Abracen, J., O'carroll, R., \& Ladha, N. (2008). Neuropsychological dysfunction in sex offenders? The Journal of Forensic Psychiatry, 2(2), 167-177. https://doi.org/10.1080/09585189108407646

Adjorlolo, S., \& Egbenya, D. L. (2016). Executive functioning profiles of adult and juvenile male sexual offenders: A systematic review. The Journal of Forensic Psychiatry \& Psychology, 27(3), 349-375. https://doi.org/10.1080/14789949.2016.1141431

Azizian, A., Hutton, S., Hughes, D., \& Sreenivasan, S. (2016). Cognitional impairment: Is there a role for cognitive assessment in the treatment of individuals civilly committed pursuant to the sexually violent predator act? Sexual Abuse: A Journal of Research and Treatment, 28(8), 755769. https://doi.org/10.1177/1079063215570757

Becerra-García, J. A., \& Egan, V. (2014). Neurocognitive functioning and subtypes of child molesters: poorer working memory differentiates incestuous from non-incestuous offenders. Psychiatry, Psychology and Law, 21(4), 585-590. https://doi.org/10.1080/13218719.2013.873974

Bechara, A., Tranel, D., \& Damasio, H. (2000). Characterization of the decision-making deficit of patients with ventromedial prefrontal cortex lesions. Brain. https://doi.org/10.1093/brain/123.11.2189

Bennett, G. K., Seashore, H. G., \& Wesman, A. G. (1982). Differential aptitude tests (Forms V and W). New York: The Psychological Corporation.

Blanchard, R., Christensen, B. K., Strong, S. M., Cantor, J. M., Kuban, M. E., Klassen, P., ... Blak, T. (2002). Retrospective self-reports of childhood accidents causing unconsciousness in phallometrically diagnosed pedophiles. Archives of Sexual Behavior, 31(6), 511-526. https://doi.org/10.1023/A:1020659331965

Blanchard, R., Kolla, N. J., Cantor, J. M., Klassen, P. E., Dickey, R., Kuban, M. E., \& Blak, T. (2007). IQ, handedness, and pedophilia in adult male patients stratified by referral source. Sexual Abuse: A Journal of Research and Treatment, 19, 285-309. https://doi.org/10.1007/s11194-007-9049-0 
Blum, K., Braverman, E. R., Holder, J. M., Lubar, J. F., Monastra, V. J., Miller, D., ... Comings, D. E. (2000). The reward deficicency syndrome: A biogenetic model for the diagnosis and treatment of impulsive, addictive, and compulsive behaviors. Journal of Psychoactive Drugs, 32(Suppl 1), 1112. https://doi.org/10.1080/02791072.2000.10736099

Brand, M., Fujiwara, E., Borsutzky, S., Kalbe, E., Kessler, J., \& Markowitsch, H. J. (2005). Decisionmaking deficits of Korsakoff patients in a new gambling task with explicit rules: Associations with executive functions. Neuropsychology. https://doi.org/10.1037/0894-4105.19.3.267

Brazil, I., van Dongen, J. D. M., Maes, J. H. R., Mars, R. B., \& Baskin-Sommers, A. R. (2018). Classification and treatment of antisocial individuals: From behavior to biocognition. Neuroscience and Biobehavioral Reviews, 91, 259-277. https://doi.org/10.1016/j.neubiorev.2016.10/010

Cantor, J. M., \& Blanchard, R. (2012). White matter volumes in pedophiles, hebephiles, and teleiophiles. Archives of Sexual Behavior. https://doi.org/10.1007/s10508-012-9954-2

Cantor, J. M., Blanchard, R., Christensen, B. K., Dickey, R., Klassen, P. E., Beckstead, A. L., ... Kuban, M. E. (2004). Intelligence, memory, and handedness in pedophilia. Neuropsychology, 18, 3-14. https://doi.org/10.1037/0894-4105.18.1.3

Cantor, J. M., Blanchard, R., Robichaud, L. K., \& Christensen, B. K. (2005). Quantitative reanalysis of aggregate data on IQ in sexual offenders. Psychological Bulletin, 131(4), 555-568. https://doi.org/10.1037/0033-2909.131.4.555

Cantor, J. M., Kabani, N., Christensen, B. K., Zipursky, R. B., Barbaree, H. E., Dickey, R., ... Blanchard, R. (2008). Cerebral white matter deficiencies in pedophilic men. Journal of Psychiatric Research, 42, 167-183. https://doi.org/10.1016/j.jpsychires.2007.10.013

Cantor, J. M., Klassen, P. E., Dickey, R., Christensen, B. K., Kuban, M. E., Blak, T., ... Blanchard, R. (2005). Handedness in pedophilia and hebephilia. Archives of Sexual Behavior, 34(4), 447-459. https://doi.org/10.1007/s10508-005-4344-7

Cantor, J. M., Kuban, M. E., Blak, T., Klassen, P. E., Dickey, R., \& Blanchard, R. (2006). Grade failure and special education placement in sexual offenders' educational histories. Archives of Sexual Behavior, 35(6), 743-751. https://doi.org/10.1007/s10508-006-9018-6 
Chun, M. M., Golomb, J. D., \& Turk-Browne, N. B. (2011). A Taxonomy of External and Internal Attention. Annual Review of Psychology, 62(1), 73-101. https://doi.org/10.1146/annurev.psych.093008.100427

Cohen, L. J., Nesci, C., Steinfeld, M., Haeri, S., \& Galynker, I. (2010). Investigating the relationship between sexual and chemical addictions by comparing executive function in pedophiles, opiate addicts and healthy controls. Journal of Psychiatric Practices, 16, 405-412. https://doi.org/10.1097/01.pra.0000390759.04581.7c.

Cohen, L. J., Nikiforov, K., Gans, S., Poznansky, O., McGeoch, P., Weaver, C., ... Galynker, I. (2002). Heterosexual male perpetrators of childhood sexual abuse: A preliminary neuropsychiatric model. Psychiatric Quarterly, 73(4), 313-336. https://doi.org/10.1023/A:1020416101092

Delis, D., Kaplan, E., \& Kramer, J. (2001). Delis Kaplan Executive Function System, examiner's manual. San Antonio, TX: The Psychological Corporation.

Eastvold, A., Suchy, Y., \& Strassberg, D. (2011). Executive function profiles of pedophilic and nonpedophilic child molesters. Journal of the International Neuropsychological Society, 17(2), 295-307. https://doi.org/10.1017/s1355617710001669

Golden, C. J. (1980). Luria-Nebraska neuropsychological battery: Manual. Los Angeles, CA: Western Psychological Services.

Hanson, R. K., \& Morton-Bourgon, K. E. (2005). The characteristics of persistent sexual offenders: A meta-analysis of recidivism studies. Journal of Consulting and Clinical Psychology. https://doi.org/10.1037/0022-006X.73.6.1154

Heaton, R. K. (1981). Wisconsin Card Sorting Test manual. Odessa, FL: Psychological Assessment Resources.

Hoaken, P. N. S., Allaby, D. B., \& Earle, J. (2007). Executive cognitive functioning and the recognition of facial expressions of emotion in incarcerated violent offenders, non-violent offenders, and controls. Aggressive Behavior, 33(5), 412-421. https://doi.org/10.1002/ab.20194

Joyal, C. C., Beaulieu-Plante, J., \& de Chantérac, A. (2014). The neuropsychology of sex offenders: A Meta-analysis. Sexual Abuse: A Journal of Research and Treatment, 26(2), 149-177. 


\section{NEUROPSYCHOLOGY OF CHILD SEXUAL OFFENDING}

https://doi.org/10.1177/1079063213482842

Joyal, C. C., Black, D. N., \& Dassylva, B. (2007). The neuropsychology and neurology of sexual deviance: A review and pilot study. Sexual Abuse: A Journal of Research and Treatment, 19(2), 155-173. https://doi.org/10.1007/s11194-007-9045-4

Kärgel, C., Massau, C., Weiß, S., Walter, M., Borchardt, V., Krueger, T. H. C., ... Schiffer, B. (2017). Evidence for superior neurobiological and behavioral inhibitory control abilities in non-offending as compared to offending pedophiles. Human Brain Mapping, 38, 1092-1104. https://doi.org/10.1002/hbm.23443

Kringelbach, M. L., \& Rolls, E. T. (2004). The functional neuroanatomy of the human orbitofrontal cortex: Evidence from neuroimaging and neuropsychology. Progress in Neurobiology, 72(5), 341-372. https://doi.org/10.1016/j.pneurobio.2004.03.006

Kruger, T. H. C., \& Schiffer, B. (2011). Neurocognitive and personality factors in homo- and heterosexual pedophiles and controls. Journal of Sexual Medicine, 8, 1650-1659. https://doi.org/10.1111/j.1743-6109.2009.01564.x

Langevin, R., \& Curnoe, S. (2008). Assessing neuropsychological impairment among sex offenders and paraphilics. Journal of Forensic Psychology Practice, 8(2), 150-173. https://doi.org/10.1080/15228930801963960

Langevin, R., Wortzman, G., Dickey, R., Wright, P., \& Handy, L. (1988). Neuropsychological impairment in incest offenders. Sexual Abuse: A Journal of Research and Treatment, 1, 401-415. https://doi.org/10.1177/107906328800100304

Langevin, R., Wortzman, G., Wright, P., \& Handy, L. (1989). Studies of brain damage and dysfunction in sex offenders. Sexual Abuse: A Journal of Research and Treatment, 2, 163-179. https://doi.org/10.1177/107906328900200204

Maniglio, R. (2009). The impact of child sexual abuse on health: A systematic review of reviews. Clinical Psychology Review, 29(7), 647-657. https://doi.org/10.1016/j.cpr.2009.08.003

Mann, R. E., Hanson, R. K., \& Thornton, D. (2010). Assessing risk for sexual recidivism: Some proposals on the nature of psychologically meaningful risk factors. Sexual Abuse: Journal of Research and Treatment. https://doi.org/10.1177/1079063210366039 


\section{NEUROPSYCHOLOGY OF CHILD SEXUAL OFFENDING}

Massau, C., Tenbergen, G., Kärgel, C., Weiß, S., Gerwinn, H., Pohl, A., ... Schiffer, B. (2017). Executive functioning in pedophilia and child sexual offending. Journal of the International Neuropsychological Society, 23, 460-470. https://doi.org/10.1017/S1355617717000315

Mohnke, S., Müller, S., Amelung, T., Krüger, T. H. C., Ponseti, J., Schiffer, B., ... Walter, H. (2014). Brain alterations in paedophilia: A critical review. Progress in Neurobiology, 122, 1-23. https://doi.org/10.1016/j.pneurobio.2014.07.005

Montague, P. R., Dolan, R. J., Friston, K. J., \& Dayan, P. (2012). Computational psychiatry. Trends in Cognitive Sciences, 16(5), 72-80. https://doi.org/10.1016/j.tics.2011.11.018

Ogilvie, J. M., Stewart, A. L., Chan, R. C. K., \& Shum, D. H. K. (2011). Neuropsychological measures of executive function and antisocial behavior: A meta-analysis. Criminology, 49(4), 1063-1107. https://doi.org/10.1111/j.1745-9125.2011.00252.x

Pacific Psychological Assessment Corporation(2004). The not real people stimulus set for assessment of sexual interest. Victoria, British Columbia, Canada: Author.

Pereda, N., Guillera, G., Forns, M., \& Gomez-Benito, J. (2009). The prevalence of child sexual abuse in community and student samples: A meta-analysis. Clinical Psychology Review. https://doi.org/10.1016/j.cpr.2009.02.007

Poeppl, T. B., Nitschke, J., Santtila, P., Schecklmann, M., Langguth, B., Greenlee, M. W., ... Mokros, A. (2013). Association between brain structure and phenotypic characteristics in pedophilia. Journal of Psychiatric Research, 47, 678-685. https://doi.org/10.1016/j.jpsychires.2013.01.003

Prigatano, G. (1978). Wechsler memory scale: A selective review of the literature. Journal of Clinical Pscyhology, 34(4), 816-832.

Randolph, C. (1998). Repeatable battery for the assessment of neuropsychological status. San Antonio, TX: Pearson.

Reitan, R. M., \& Wolfson, D. (1985). The Halstead-Reitan neuropsychological test battery: Theory and clinical implication. Tucson, AZ: Neuropsychology Press.

Robertiello, G., \& Terry, K. J. (2007). Can we profile sex offenders? A review of sex offender typologies. Aggression and Violent Behavior, 12(5), 508-518. https://doi.org/10.1016/j.avb.2007.02.010 


\section{NEUROPSYCHOLOGY OF CHILD SEXUAL OFFENDING}

Rolls, E. T. (2000). The orbitofrontal cortex and reward. Cerebral Cortex, 10(3), 284-294. https://doi.org/10.1093/cercor/10.3.284

Roszyk, A., \& Łukaszewska, B. (2011). The neuropsychological determinants of sexual crimes. Acta Neuropsychologica, 9(4), 369-381.

Russell, E. W., Russell, S. L. K., \& Hill, B. D. (2005). The fundamental psychometric status of neuropsychological batteries. Archives of Clinical Neuropsychology, 20, 785-794.

Schiffer, B., Peschel, T., Paul, T., Gizewski, E., Forsting, M., Leygraf, N., ... Krueger, T. H. C. (2007). Structural brain abnormalities in the frontostriatal system and cerebellum in pedophilia. Journal of Psychiatric Research, 41(9), 753-762.

https://doi.org/10.1016/j.jpsychires.2006.06.003

Schiffer, B., \& Vonlaufen, C. (2011). Executive dysfunctions in pedophilic and nonpedophilic child molesters. Journal of Sexual Medicine, 8(7), 1975-1984. https://doi.org/10.1111/j.17436109.2010.02140.x

Schiltz, K., Witzel, J., Northoff, G., Zierhut, K., Gubka, U., Fellmann, H., ... Bogerts, B. (2007). Brain pathology in pedophilic offenders: Evidence of volume reduction in the right amygdala and related diencephalic structures. Archives of General Psychiatry, 64(6), 737-746.

Scott, M. L., Cole, J. K., McKay, S. E., Golden, C. J., \& Liggett, K. R. (1984). Neuropsychological Performance of Sexual Assaulters and Pedophiles. Journal of Forensic Sciences, JFSCA.

Seto, M. C. (2008). Pedophilia and sexual offending against children. Theory, assessment, and intervention. Washington, DC: American Psychological Association.

Spinella, M., \& White, J. (2006). Neuroanatomical substrates for sex offenses. International Journal of Forensic Psychology, 1(3), 84-94.

Stephan, K. E., \& Mathys, C. (2014). Computational approaches to psychiatry. Current Opinion in Neurobiology, 25, 85-92. https://doi.org/10.1016/j.conb.2013.12.007

Suchy, Y. (2009). Executive functioning: Overview, assessment, and research issues for nonneuropsychologists. Annals of Behavioral Medicine, 37(2), 106-116. https://doi.org/10.1007/s12160-009-9097-4

Suchy, Y., Eastvold, A. D., Strassberg, D. S., \& Franchow, E. I. (2014). Understanding processing 
speed weaknesses among pedophilic child molesters: Response style vs. neuropathology. Journal of Abnormal Psychology, 123(1), 273-285. https://doi.org/10.1037/a0035812

Suchy, Y., Whittaker, J. W., Strassberg, D. S., \& Eastvold, A. (2009a). Neurocognitive differences between pedophilic and nonpedophilic child molesters. Journal of the International Neuropsychological Society, 15(2), 248-257. https://doi.org/10.1017/S1355617709090353

Suchy, Y., Whittaker, W. J., Strassberg, D. S., \& Eastvold, A. (2009b). Facial and prosodic affect recognition among pedophilic and nonpedophilic criminal child molesters. Sexual Abuse : A Journal of Research and Treatment, 21(1), 93-110. https://doi.org/10.1177/1079063208326930

Thakker, J., \& Ward, T. (2012). An integrated theory of sexual reoffending. Psychiatry, Psychology and Law. https://doi.org/10.1080/13218719.2011.561765

Turner, D., Laier, C., Brand, M., Bockshammer, T., Welsch, R., \& Rettenberger, M. (2018). Response inhibition and impulsive decision-making in sexual offenders against children. Journal of Abnormal Psychology. https://doi.org/10.1037/abn0000359

Ward, T., \& Beech, A. (2006). An integrated theory of sexual offending. Aggression and Violent Behavior, 11(1), 44-63. https://doi.org/10.1016/j.avb.2005.05.002

Ward, T., \& Beech, A. R. (2017). The integrated theory of sexual offending-revised. In D. P. Boer (Ed.), The Wiley Handbook on the Theories, Assessment and Treatment of Sexual Offending. John Wiley \& Sons, Ltd. https://doi.org/10.1002/9781118574003.wattso006

Ward, T., \& Fortune, C. A. (2016). From dynamic risk factors to causal processes: a methodological framework. Psychology, Crime and Law. https://doi.org/10.1080/1068316X.2015.1117080

Ward, T., Polaschek, D. L. L., \& Beech, A. R. (2006). Theories of Sexual Offending. Chichester: John Wiley \& Sons, Ltd.

Wechsler, D. (1945). Wechsler memory scale. San Antonio, TX: Psychological Corporation.

Wood, R. L., \& Liossi, C. (2006). Neuropsychological and Neurobehavioral Correlates of Aggression Following Traumatic Brain Injury. Journal of Neuropsychiatry. https://doi.org/10.1176/appi.neuropsych.18.3.333

Young, M. H., Justice, J., \& Erdberg, P. (2012). A comparison of rape and molest offenders in prison psychiatric treatment. International Journal of Offender Therapy and Comparative Criminology, 
NEUROPSYCHOLOGY OF CHILD SEXUAL OFFENDING

56(7), 1103-1123. https://doi.org/10.1177/0306624X11417361 
Table 1

The Characteristics and Internal Validity Threats of the Studies on Child Sexual Offenders

\begin{tabular}{|c|c|c|c|}
\hline Study & Participants & Measures & Internal validity threats and quality rating \\
\hline $\begin{array}{l}\text { Young et al. } \\
\text { (2012) }\end{array}$ & $\begin{array}{l}\text { Comparison of CSOs } \\
(n=15)\end{array}$ & $\begin{array}{l}\text { (1) HR test battery } \\
\text { (2) WCST }\end{array}$ & $\begin{array}{l}\text { - Uncertain representativeness } \\
\text { - } \quad \text { No sample size calculation given and a small sample size used } \\
\text { - } \quad \text { No exclusion or matching criteria reported, but normative data } \\
\quad \text { were adjusted for age and education level } \\
\text { Quality rating: fair }\end{array}$ \\
\hline $\begin{array}{l}\text { Langevin and } \\
\text { Curnoe (2008) }\end{array}$ & $\begin{array}{l}\text { Comparison of CSOs } \\
(n=843) \text { with } \\
\text { normative data. }\end{array}$ & HR (impairment index) & $\begin{array}{l}\text { - No sample size calculation given } \\
\text { - No exclusion or matching criteria reported, but age and IQ were } \\
\text { statistically controlled for } \\
\text { Quality rating: good }\end{array}$ \\
\hline $\begin{array}{l}\text { Scott et al. } \\
(1984)\end{array}$ & $\begin{array}{l}\text { Comparison of CSOs } \\
(n=14) \text { with normative } \\
\text { data. }\end{array}$ & Luria battery & $\begin{array}{l}\text { - Uncertain representativeness } \\
\text { - No sample size calculation given and a small sample size used } \\
\text { - } \\
\text { No exclusion or matching criteria reported } \\
\text { - No statistical control of relevant confounders, but normative } \\
\text { data were adjusted for age and education level } \\
\text { Quality rating: fair }\end{array}$ \\
\hline
\end{tabular}



Abracen et al.
12 CSOs vs. 12
(1) TMT B
(2008)
nonviolent OC vs. 13
(2) Progressive Matrices
$\mathrm{HC}$.
(3) Williams Verbal Learning Test

Joyal et al.
Comparison of CSOs
(1) Stroop
( $n=12)$ with normative
(2) COWAT data.
(3) CVLT
(4) WCST
(5) TMT B
(6) $\mathrm{ROCF}$

(2007)

Turner et al. CSOs vs. HC.

(2018)

\section{(1) Go/No-Go task (56 CSOs vs. 63 - Uncertain representativeness} $\mathrm{HC})$

(2) Modified IGT (63 CSOs vs. 63 $\mathrm{HC})$

(3) Modified GDT (59 CSOs vs. $63 \mathrm{HC})$. controlled for

Quality assessment: good
- Uncertain representativeness

- No sample size calculation given and a small sample size used.

- No matching criteria reported, but relevant confounders were statistically controlled for.

Quality rating: fair

- No sample size calculation given and a small sample size used

- No exclusion or matching criteria reported

- No statistical control of relevant confounders, but normative data of similar age and education level were used

Quality assessment: fair

- No sample size calculation given

- No matching criteria reported, but age and IQ were statistically

Note. CSOs = child sexual offenders; OC = offender controls; HC = healthy controls; HR = Halstead-Reitan test battery; WCST = Wisconsin Card Sorting Test; TPT Total Time $=$ Tactual Performance Test - Total Time $;$ TPT Memory $=$ Tactual Performance Test - Memory; TPT Location $=$ Tactual Performance Test - Location; TMT $=$ Trail Making Test; COWAT = Controlled Oral Word Association Task; CVLT = California Verbal Learning Test; ROCF = Rey - Osterrieth Complex Figure; IGT = Iowa Gambling Task; GDT = Game of Dice Task. 
Table 2

The Characteristics, and Internal Validity Threats of the Studies on Subgroups of Child Sexual Offenders

\begin{tabular}{|c|c|c|c|}
\hline Study & Participants & Measures & Internal validity threats and quality rating \\
\hline $\begin{array}{l}\text { Suchy et } \\
\text { al.(2009a) }\end{array}$ & $\begin{array}{l}20 \text { pd CSOs vs. } 20 \\
\text { nonpd CSOs vs. } 20 \\
\text { HC. }\end{array}$ & $\begin{array}{l}6 \text { composite scores: } \\
\text { (1) Semantic knowledge, measured by WAIS-III Information } \\
\text { subtest, Peabody Individual Achievement Test-Reading } \\
\text { comprehension subtest, and Recognition vocabulary } \\
\text { (2) Executive functions, measured by Stroop, Ruff Figural } \\
\text { Fluency Test, and Behavioral Dyscontrol Scale } \\
\text { (3) Auditory Memory, measured by WMS Logical Memory } \\
\text { subtest } \\
\text { (4) Visual Memory, measured by WMS Visual reproduction } \\
\text { subtest } \\
\text { (5) Processing speed and (6) Motor Speed, measured by Simple } \\
\text { choice reaction time and Complex choice reaction time task }\end{array}$ & $\begin{array}{l}\text { - Uncertain representativeness } \\
\text { - } \quad \text { No sample size calculation given and } \\
\text { relatively small sample sizes used } \\
\text { - No matching criteria reported, but age and IQ } \\
\text { were statistically controlled for } \\
\text { Quality rating: good }\end{array}$ \\
\hline $\begin{array}{l}\text { Schiffer } \\
\text { and }\end{array}$ & $\begin{array}{l}15 \text { pd CSOs vs. } 15 \\
\text { nonpd CSOs vs. } 16\end{array}$ & $\begin{array}{l}\text { (1) Inhibitory control, measured by Go/No-go task } \\
\text { (2) Cognitive flexibility, measured by WCST }\end{array}$ & $\begin{array}{l}\text { - No sample size calculation given and small } \\
\text { sample sizes used. }\end{array}$ \\
\hline $\begin{array}{l}\text { Vonlaufen } \\
(2011)\end{array}$ & $\begin{array}{l}\text { violent OC vs. } 17 \\
\text { HC. }\end{array}$ & $\begin{array}{l}\text { (3) Visuospatial memory, measured by CBT and WMS-R Visual } \\
\text { Reproduction task } \\
\text { (4) Verbal memory, measured by WMS-R Logical Memory task I } \\
\text { and II }\end{array}$ & $\begin{array}{l}\text { - No statistical control of relevant confounders, } \\
\text { but the groups were matched on age and } \\
\text { education level. } \\
\text { Quality rating: Good }\end{array}$ \\
\hline
\end{tabular}


(5) Verbal fluency, measured by Regensburger

Wortflüssigkeitstest

Eastvold 30 pd CSOs vs. $30 \quad 4$ composite scores:

et al. nonpd CSOs vs. 29

(2011) OC.

\author{
Kärgel et 37 nonoffending $\quad$ Go/ No-go task \\ al. (2017) paedophiles vs. 40 \\ Pd CSOs vs. $40 \mathrm{HC}$.
}

\section{Cohen et 22 pd CSOs vs. 24}

al.(2002) HC
(1) Inhibitory control, measured by DKEFS Color-Word Interference Test-Inhibition condition and DKEFS ColorWord Interference Test - Inhibition/ Switch condition

(2) Cognitive flexibility, measured by DKEFS TMT NumberLetter Switching condition and DKEFS Design Fluency test Switch condition

(3) Working memory, measured by WMS-III Digit Span Backwards and WMS-III Spatial Span Backwards

(4) Attention, measured by WMS-III Digit Span total forward score and WMS-III Spatial Span total forward score

Various tests, combined into 4 neuropsychological functions:

(1) Category formation and set switching, measured by TMT B and WCST

(2) Attention, measured by Stroop and TMT A
- Uncertain representativeness

- No sample size calculation given

- In contrast to other relevant confounders, age was not statistically controlled for, but groups did not differ in age

Quality rating: good
- Uncertain representativeness

- No sample size calculation given Quality rating: Good

- Uncertain representativeness .

- No sample size calculation given and relatively sample sizes used 
(3) Verbal functions, measured by COWAT and WAIS-R Vocabulary

(4) Impulsivity, measured by Gambling Task
- In contrast to education level, age was not statistically controlled for, but groups did not differ in age.

Quality rating: good

\section{Cohen et}

Pd CSOs vs. OADD

al. (2010) vs. HC.
(1) Impulsivity, measured by MFFT (24 pd CSOs vs. 23 OADD vs. $24 \mathrm{HC}$ ) and Porteus Mazes (24 pd CSOs vs. 15 OADD vs. $11 \mathrm{HC})$.

(2) Cognitive flexibility, measured by WCST (22 pd CSOs vs. 20 OADD vs. $22 \mathrm{HC}$ ) and TMT B (49 pd CSOs vs. 19 OADD vs. $49 \mathrm{HC})$

(3) Attention, measured by Stroop Color-Word Test (47 pd CSOs vs. 50 OADD vs. $80 \mathrm{HC}$ ) and TMT A (50 pd CSOs vs. 21 OADD vs. 49 HC)

(4) Verbal fluency, measured by COWAT

\section{Massau et 45 Pd CSOs vs. 45}

al. (2017) nonoffending paedophiles vs. 19

Nonpd CSOs vs. 49

HC.
Five subtests of the CANTAB, assessing impulsivity, cognitive flexibility, and working memory: SST; IST; IED, SOC, and SWM
Uncertain representativeness

No sample size calculation given and (relatively) small sample sizes used in a number of the analyses

- Invalid method to diagnose paedophilia (i.e., to assess group membership)

No matching criteria reported and groups differed on age, education, and gender. Only education was statistically controlled for, but age and gender did not correlate with neuropsychological performance in this study Quality rating: Poor

- Uncertain .representativeness

- No sample size calculation given Quality rating: Good 


\section{Kruger 20 pd CSOs vs. 24 (1) Cognitive flexibility measured by WCST}

and

$\mathrm{HC}$

Schiffer

Azizian et Comparison of 11

al. (2016) pd CSOs with normative data.
Langevin Comparison of pd

et al. CSOs with

(1989) normative data \&

\section{Comparison of}

incestual CSOs with

normative data.
(2) Visuospatial working memory, measured by CBT

(3) Attention and concentration, measured by d2 AttentionDeficit Test
The RBANS yields 5 index scores on the following neuropsychological domains: Immediate memory, Visuospatial abilities, Language, Attention, and Delayed memo
- No sample size calculation given and relatively small sample sizes used

- No matching criteria reported, but age (and education level) were statistically controlled for

Quality rating: Good

- Uncertain representativeness.

- No sample size calculation given

- No statistical control for relevant confounders, but a matched normative sample (matched for age, education level, and etnicity) used

Quality rating: Good
(1) WMS (39 pd CSOs, 48 incestual CSOs)

(2) DAP Space Relations Test (45pd CSOs, 36 incestual CSOs)
- Uncertain representativeness

- No sample size calculation given

- Psychometric limitations of the neuropsychological measures

- No exclusion or matching criteria reported. An age-corrected quotient is only computed with respect to WMS (not with respect to the DAP Space Relations Test). 
Quality rating: Poor

Suchy et 18 pd CSOs vs. 23 Affect recognition abilities, assessed by

al.(2009b) nonpd CSOs vs. 21

$\mathrm{HC}$

(1) Facial affect recognition task (total numbers of errors and response time)

(2) Prosody perception task (total number of errors)

Suchy et $20 \mathrm{pd}$ CSOs vs.20

al. (2014) nonpd CSOs vs. 20

OC vs. normative

sample.

Different domains of processing speed, assessed by

(1) Finger Tapping test from the HR battery to assess motor speed

(2) WAIS III, Symbol Search to assess visual-perceptual speed

(3) WAIS III, Digit Symbol Coding to assess visual-motor integration

Aetiology of slow processing speed, assessed by Modified ITT

$\begin{array}{ll}\text { Langevin } & \text { Comparison of } 83 \\ \text { et al. } & \text { HR battery } \\ \text { (1988) } & \text { normative data and } \\ & 14 \text { nonviolent OC. }\end{array}$

- Uncertain representativeness

- No sample size calculation given and relatively small sample sizes used.

Quality rating: Good

- Uncertain representativeness

- No sample size calculation given and relatively small sample sizes used

- When comparing CSOs to the normative sample, there was no statistical control for relevant confounders. However, the normative samples that were used, were matched for age and education

Quality rating; Good

- Uncertain representativeness

- No sample size calculation given and relatively small sample size was used

- No exclusion or matching criteria reported

- Not clear whether the normative data were adjusted for age and education level 
- Potential confounders were not statistically controlled for when comparing incestual

CSOs to OC

Quality rating: Poor

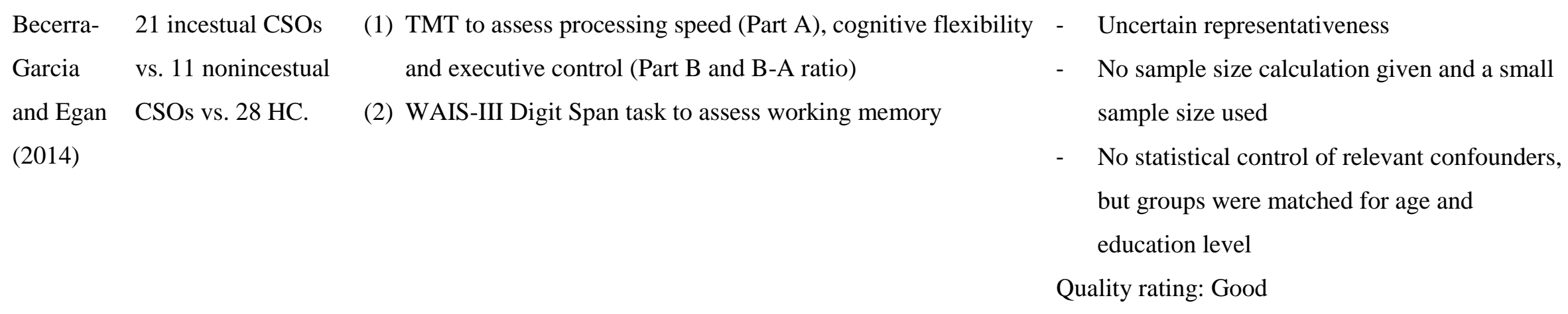

Note. CSOs = child sexual offenders; pd CSOs = paedophilic child sexual offenders; nonpd CSOs = nonpaedophilic child sexual offenders; $\mathrm{HC}=$ healthy controls; OC $=$ offender controls; OADD = opiate addicts who are detoxified; WAIS-III = Wechsler Adult Intelligence Scale (3rd ed.); WMS = Wechsler Memory Scale; WCST = Wisconsin Card Sorting Test CBT $=$ Corsi Block Tapping Test; WMS-R $=$ Wechsler Memory Scale Revised; DKEFS = Delis Kaplan Executive Function Scale; WMS-III = Wechsler Memory Scale (3rd ed.); TMT = Trail Making Test; COWAT = Controlled Oral Word Association Task; WAIS-R = Wechsler Adult Intelligence Scale Revised; MFFT = Matching Familiar Figures Test; CANTAB = Cambridge Neuropsychological Test Automated Battery; SST = Stop Signal Task; IST = Information Sampling Task; IED = Intra/Extradimensional Set Shift Task; SOC = Stockings of Cambridge Task; SWM = Spatial Working Memory Task; RBANS = Repeatable Battery for the Assessment of Neuropsychological Status; DAP Space Relations Test = Differential Aptitude Space Relations Test; HR = Halstead-Reitan test battery; ITT = Visual Inspection Time Task. 


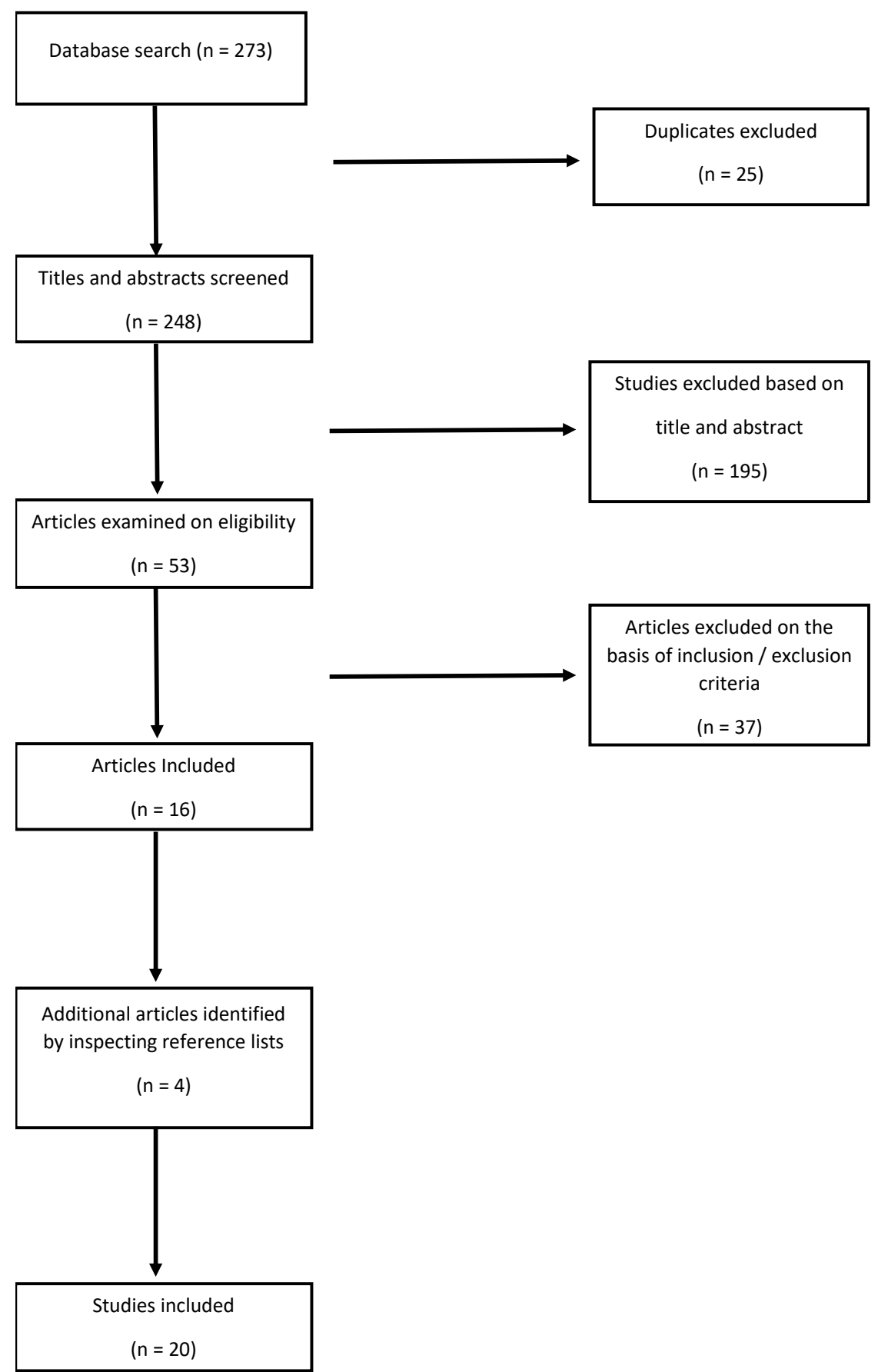

Figure 1. Flow chart of the search strategy and the selection of studies. 


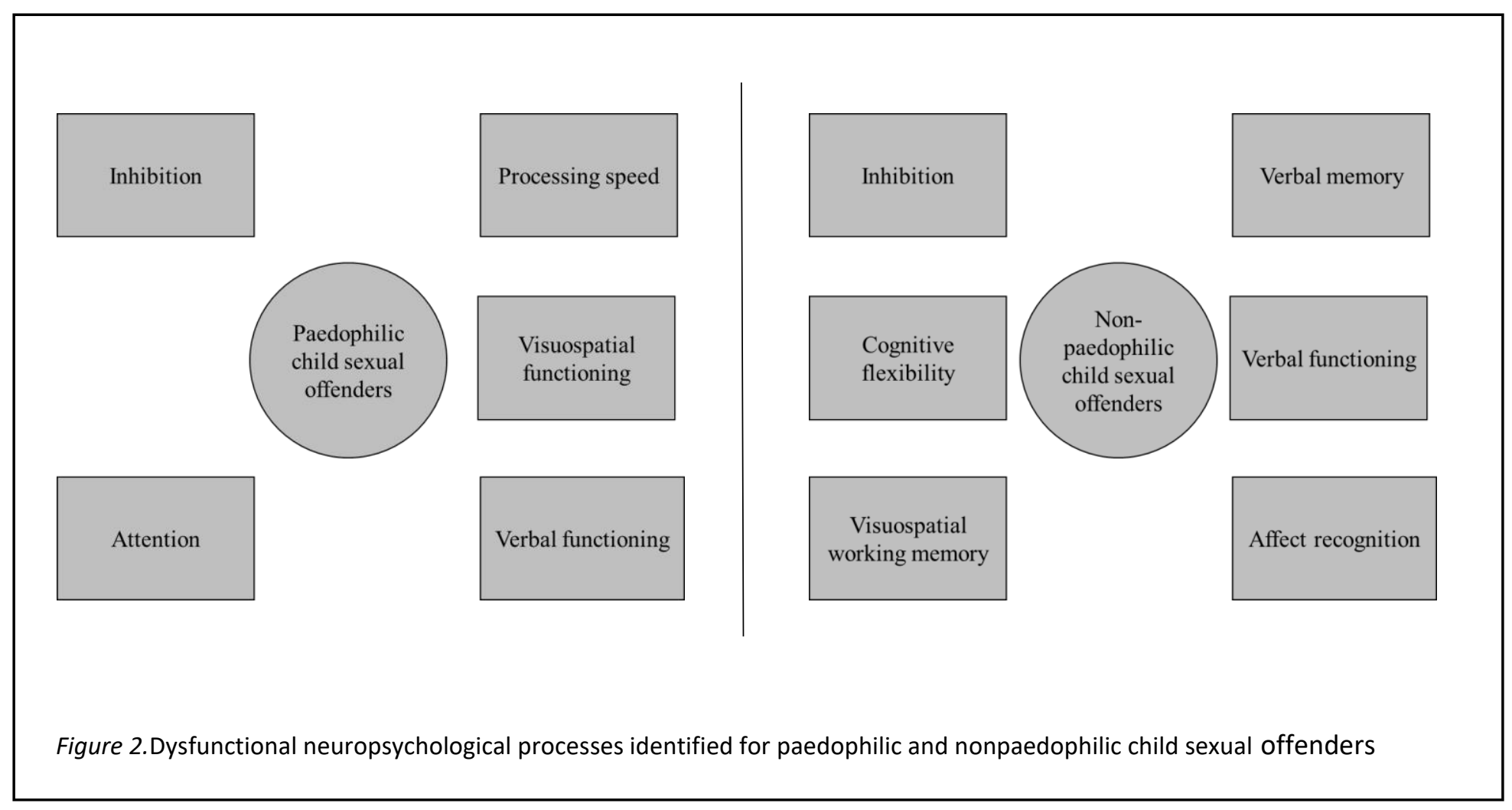

